\title{
Video Article \\ Analysis of Complex Molecules and Their Reactions on Surfaces by Means of Cluster-Induced Desorption/lonization Mass Spectrometry
}

\author{
Karolin Bomhardt ${ }^{* 1}$, Pascal Schneider* ${ }^{*}$, André Portz ${ }^{1}$, Christoph R. Gebhardt ${ }^{2}$, Michael Dürr ${ }^{1}$ \\ ${ }^{1}$ Institute of Applied Physics and Center for Materials Research (LaMa), Justus Liebig University Giessen \\ ${ }^{2}$ Bruker Daltonik GmbH \\ * These authors contributed equally
}

Correspondence to: Michael Dürr at Michael.Duerr@ap.physik.uni-giessen.de

URL: https://www.jove.com/video/60487

DOI: doi:10.3791/60487

Keywords: Chemistry, Issue 157, mass spectrometry, cluster, desorption, matrix-free, soft, surface adsorbates, reaction kinetics, H/D exchange

Date Published: 3/1/2020

Citation: Bomhardt, K., Schneider, P., Portz, A., Gebhardt, C.R., Dürr, M. Analysis of Complex Molecules and Their Reactions on Surfaces by Means of Cluster-Induced Desorption/lonization Mass Spectrometry. J. Vis. Exp. (157), e60487, doi:10.3791/60487 (2020).

\section{Abstract}

Desorption/lonization Induced by Neutral $\mathrm{SO}_{2}$ Clusters (DINeC) is employed as a very soft and efficient desorption/ionization technique for mass spectrometry (MS) of complex molecules and their reactions on surfaces. DINeC is based on a beam of $\mathrm{SO}_{2}$ clusters impacting on the sample surface at low cluster energy. During cluster-surface impact, some of the surface molecules are desorbed and ionized via dissolvation in the impacting cluster; as a result of this dissolvation-mediated desorption mechanism, low cluster energy is sufficient and the desorption process is extremely soft. Both surface adsorbates and molecules of which the surface is composed of can be analyzed. Clear and fragmentationfree spectra from complex molecules such as peptides and proteins are obtained. DINeC does not require any special sample preparation, in particular no matrix has to be applied. The method yields quantitative information on the composition of the samples; molecules at a surface coverage as low as $0.1 \%$ of a monolayer can be detected. Surface reactions such as H/D exchange or thermal decomposition can be observed in real-time and the kinetics of the reactions can be deduced. Using a pulsed nozzle for cluster beam generation, DINeC can be efficiently combined with ion trap mass spectrometry. The matrix-free and soft nature of the DINeC process in combination with the MS ${ }^{n}$ capabilities of the ion trap allows for very detailed and unambiguous analysis of the chemical composition of complex organic samples and organic adsorbates on surfaces.

\section{Video Link}

The video component of this article can be found at https://www.jove.com/video/60487/

\section{Introduction}

Surface sensitive analysis techniques are often based on particle probes such as low energy electrons, atoms, or ions which strongly interact with solid samples. As a consequence, they show high surface sensitivity and detailed information on surface structure can be obtained ${ }^{1}$. Chemical information, however, is often limited. As an example, X-ray photoelectron spectroscopy can give quantitative information on the atomic composition and on the average chemical environment of a given species (e.g., the carbon atoms in an organic molecule adsorbed on a surface ${ }^{2}$ ). However, more detailed information on complex, surface-adsorbed molecules, such as their detailed structure or binding sites, is difficult to be obtained with standard surface analysis techniques. On the other hand, the need for such information is growing with the increasing interest in surface functionalization by means of organic molecules. The expanding fields of on-surface synthesis ${ }^{3}$ or surface functionalization by attachment of biomolecules ${ }^{4,5}$ are two prominent examples. In all these fields, fundamental questions on substrate-adsorbate and adsorbateadsorbate interactions are investigated in order to better understand the systems. For these investigations, a maximum of information on the adsorbed molecules is desirable.

In part, secondary ion mass spectrometry (SIMS) can give such information. First, SIMS is highly surface sensitive. Second, as the sputtered adsorbates and their fragments are detected by means of MS, information well beyond atomic composition is obtained. Depending on the nature of the chemical species adsorbed on the surface, it can be identified by its molecular mass and fragment pattern observed in the mass spectrum $^{6}$. The fragments induced by the primary ions indeed can help for the identification of the analyzed material. On the other hand, if primary-ion induced modification (fragmentation, ion-induced reactions, mixing) of the sample is too strong, most information on the original state of the sample is lost. Thus, major efforts have been undertaken to reduce fragmentation in SIMS (e.g., using charged molecular clusters as primary ions $\left.{ }^{7,8,9}\right)$. However, fragmentation still dominates SIMS spectra of large macromolecules and biological samples ${ }^{10}$, limiting the application of SIMS in various fields.

As an alternative, we have shown desorption/ionization induced by neutral clusters (DINeC) to be a soft and matrix-free ionization method which has been successfully employed for mass spectrometric analysis of complex molecules ${ }^{11,12,13,14,15,16,17}$. DINeC is based on a beam of molecular clusters which consist of $10^{3}$ to $10^{4} \mathrm{SO}_{2}$ molecules (Figure 1). When the clusters impact on the sample, they interact in various ways with the molecules on and in the surface: first, a part of the cluster's kinetic energy is redistributed and activates desorption. Similarly 
important, the desorbing molecule is dissolved in the cluster during cluster-surface impact ${ }^{11,18,19}$ (Figure 1 and Figure 2). In other words, based on the high dipole moment of $\mathrm{SO}_{2}$, the clusters very efficiently serve as a transient matrix for polar analytes. As a result, desorption of the analyte molecules takes place at cluster energies as low as $1 \mathrm{eV} /$ molecule and below. The soft nature of the desorption process is further supported by rapid cooling of the system when the $\mathrm{SO}_{2}$ cluster shatters during and after surface impact ${ }^{11,19}$. As a consequence of these various aspects, cluster-induced desorption of complex molecules such as peptides, proteins, lipids, and dyes proceeds without any fragmentation of the desorbing molecules ${ }^{11,15}$; typical mass spectra show the dominant peak at the $\mathrm{m} / \mathrm{z}$ value of the intact molecule $\left([\mathrm{M}+\mathrm{H}]^{+}\right.$or $\left.[\mathrm{M}-\mathrm{H}]^{-}, \mathrm{Figure} 3\right)$ Depending on the number and nature of functional groups in the molecule, multiple charged cations of the form [M $+n \cdot H]^{n+}$ are observed ${ }^{11,15,18}$ For biomolecules, ionization typically takes place via uptake or abstraction of a proton at a basic or acidic functional group, respectively ${ }^{11}$. If water molecules are present in the sample, $\mathrm{SO}_{2}$ molecules from the cluster can react with these water molecules forming sulfurous acid ${ }^{18}$. The latter can act as an efficient proton source which further promotes the ionization process in case of ionization via proton uptake (positive ion mode $)^{13,18}$.

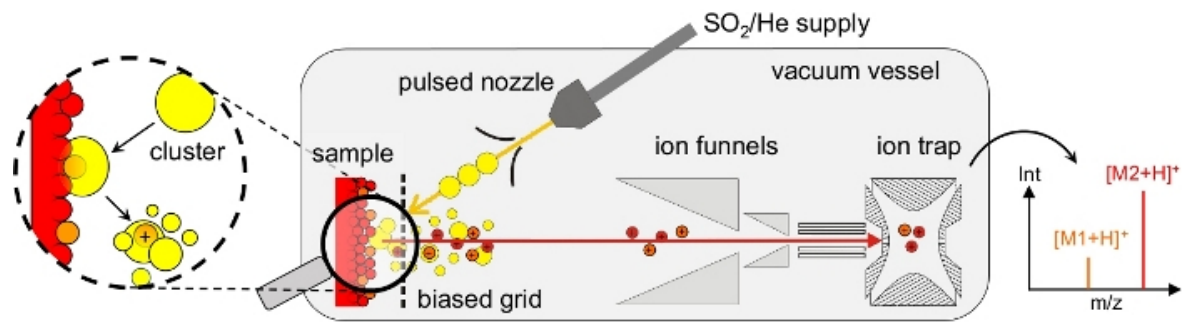

Figure 1: Schematic illustration of cluster-induced desorption/ionization and experimental set-up. Cluster-induced desorption/ionization is performed in a high-vacuum vessel. A beam of $\mathrm{SO}_{2}$ clusters (yellow dots) is produced via supersonic expansion of a $\mathrm{SO}_{2} / \mathrm{He}$ gas mixture from a pulsed nozzle. During cluster-surface impact, surface molecules are desorbed and ionized. Molecular ions (red/orange dots) are transferred via a biased grid, a dual ion funnel inlet, and octopolar ion guides into the ion trap for mass spectrometry. Typical mass spectra show dominant peaks at $m / z$ values of the intact molecules, here: M1 (orange) and M2 (red) in positive ion mode. Blow up: During cluster surface impact, the desorbed

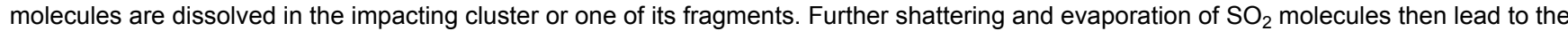
bare, intact molecular ion as detected in the mass spectrometer. See also Figure 2. Please click here to view a larger version of this figure.

(A)

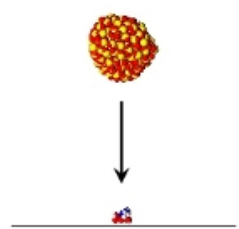

(B)

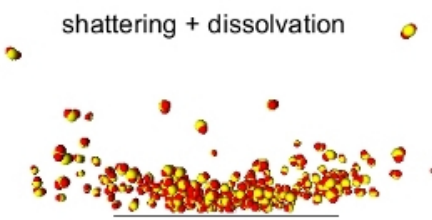

(C)

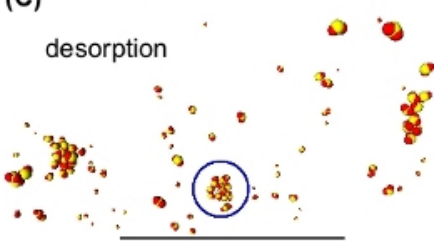

Figure 2: Snapshots of molecular dynamics simulations illustrating cluster-induced desorption via dissolvation. (A) An $\mathrm{SO}_{2}$ cluster (300 molecules) approaches the surface with $1250 \mathrm{~m} / \mathrm{s}$ perpendicular to the surface on which a dipeptide (aspartic acid-arginine, ASP-ARG) is

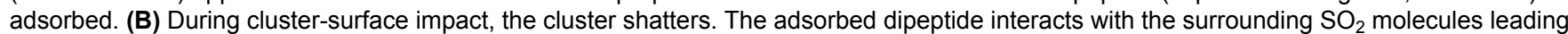
to its dissolvation in one of the cluster fragments. (C) The cluster fragments are repelled from the surface. The labelled fragment (blue circle) carries the dipeptide which is desorbed in this fragment. This figure has been modified from reference 19. Please click here to view a larger version of this figure.

(A)

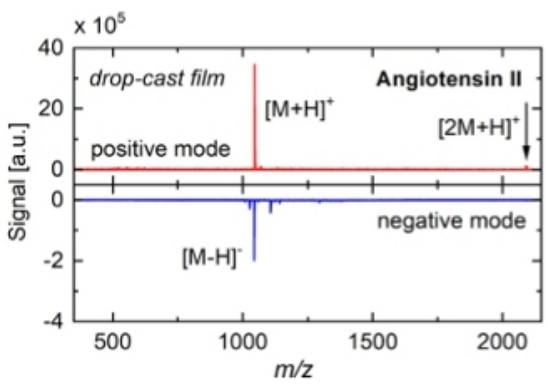

(B)

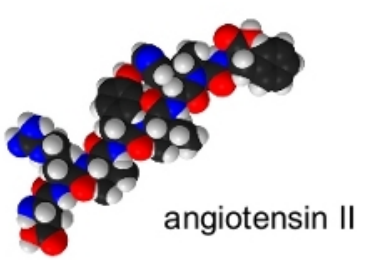

$\mathrm{H}_{2} \mathrm{~N}$-Asp-Arg-Val-Tyr-Ile-His-Pro-Phe- $\mathrm{COOH}$

Figure 3: Representative mass spectrum and molecular model of angiotensin II. (A) Mass spectra (top panel: positive ion mode, bottom panel: negative ion mode) as obtained after cluster-induced desorption/ionization from an angiotensin II sample. The sample was prepared by drop-casting the respective solution on a Si wafer (covered by its natural oxide). The main peaks are assigned to the intact biomolecule, $[\mathrm{M}+\mathrm{H}]^{+}$ and $[\mathrm{M}-\mathrm{H}]^{-}$; no fragmentation patterns are observed. Dimers $\left([2 \mathrm{M}+\mathrm{H}]^{+}\right.$, arrow) further indicate the soft nature of the desorption process. The positive ion signal is more intense due to the influence of the $\mathrm{SO}_{2}$ clusters ${ }^{18}$. (B) Space-filling model and amino acid sequence of angiotensin II. White balls indicate hydrogen atoms; black: carbon; blue: nitrogen; red: oxygen. Please click here to view a larger version of this figure.

$\mathrm{DINeC}$ can be applied to any sort of solid sample which is compatible with high-vacuum conditions. No special sample preparation is required, in particular no matrix has to be applied prior to DINeC-MS measurements, in contrast to matrix-assisted laser desorption/ionization (MALDI) mass spectrometry and related techniques ${ }^{20,21}$. This enables real time measurements of chemical changes of the sample with varying experimental conditions such as background pressure of reactive species in the vacuum chamber ${ }^{22}$ or sample temperature. The detection limit of DINeC-MS has been shown to be in the femtomole range ${ }^{11}$. When applied to the analysis of biomolecules adsorbed on solid surfaces in the submonolayer regime, a surface coverage as low as $0.1 \%$ of a monolayer was detected ${ }^{23}$. In this coverage regime, the signal intensity depends linearly on 
surface coverage and DINeC-MS can be used for quantitative analysis of the surface composition ${ }^{23}$. In the case of mixed samples, a quantitative evaluation of the sample composition is possible ${ }^{17,24}$, as no major effect of the chemical environment on the ionization probability is observed (e.g., in the case of mixed lipid/peptide samples ${ }^{17}$ ). This is in clear contrast to SIMS, for which the ionization probability of a given species is typically strongly influenced by the presence of different chemical components (the so-called "matrix effect"25,26).

In addition to surface analysis, chemical composition in the subsurface region can be probed by means of depth profiling ${ }^{17}$. With the current setup, typical desorption rates of cluster-induced desorption of biomolecules are of the order $10^{-3} \mathrm{~nm} / \mathrm{s}$. A high depth resolution in the range of 1 to 2 $\mathrm{nm}$ has been observed for mixed lipid/peptide samples ${ }^{17}$.

A further field of application is the combination of DINeC-MS with thin layer chromatography (TLC). Conventional TLC plates can be directly analyzed by means of DINeC-MS. Position-dependent mass spectra can be acquired from the TLC plates and thus mass-specific chromatograms can be obtained from the TLC plates ${ }^{27}$. No re-elution of the separated analytes is necessary, different to TLC in combination with $\mathrm{ESI}^{28,29}$. No matrix is needed for the DINeC-MS + TLC combination either, in contrast to the coupling of TLC with MALDI ${ }^{28,29}$.

Desorption electrospray ionization (DESI) is also a soft desorption/ionization method for MS-applications ${ }^{30,31}$. The most striking differences between DINeC and DESI are: the quantitative nature of DINeC ${ }^{23}$, its compatibility with ultra-high-vacuum (UHV) conditions, in particular the possibility to investigate samples prepared and transferred in UHV conditions without breaking the vacuum ${ }^{23}$, as well as the possibility to efficiently desorb nonpolar molecules ${ }^{19}$.

In principle, DINeC as desorption/ionization source can be coupled to any type of mass spectrometer. However, the combination with ion trap mass spectrometry features two main advantages: first, the pulse width and repetition rate of a typical pulsed cluster beam correspond very well to the discontinuous accumulation time as well as the spectral rate of the ion trap ${ }^{15,32}$. Second, the soft nature of the DINeC process leads to desorption of intact molecules. In combination with the $\mathrm{MS}^{\mathrm{n}}$ capabilities of ion trap mass spectrometry, this allows for a most comprehensive analysis of the investigated samples ${ }^{15}$.

\section{Protocol}

NOTE: The protocol can be paused at any time.

\section{Preparation of substrates}

1. For standard samples, cut the substrates from silicon wafers (thickness of approx. 0.5 to $1 \mathrm{~mm}$ ) in pieces of $1 \times 1 \mathrm{~cm}^{2}$.

2. Clean the Si substrates in an ultrasound bath of ethanol and acetone for 15 min each.

3. Dry the substrates in a stream of dry nitrogen gas.

\section{Preparation of samples}

\section{Standard sample preparation}

1. For standard samples, prepare the solution containing the analyte molecules according to the scientific question to be addressed. Concentration of the analyte should be at least $1 \times 10^{-10} \mathrm{~mol} / \mathrm{L}$.

2. Drop-cast 5 to $30 \mu \mathrm{L}$ of the sample solution on the substrate. Depending on vapor pressure of the solvent, let the sample dry under ambient conditions or in a desiccator until all solvent has been evaporated and a dry film has been formed. Depending on the amount of substance applied, the thickness of the film can be between several ten $\mu \mathrm{m}$ (visual inspection possible) and the monolayer to submonolayer regime (thus not detectable by eye).

3. Mount the samples on the sample holder (e.g., using sticky tape or clamps tightened by screws, depending on the samples and vacuum conditions required).

4. If possible, additionally mount a reference sample such as a micrometer-thick film of angiotensin II, on the sample holder.

\section{Alternative sample preparation}

1. Use alternative sample preparation schemes such as electrospray ion-beam deposition (ES-IBD) in vacuum or dip coating in a respective solution if applicable.

2. Mount samples which are to be prepared and transferred in vacuum on the DINeC sample holder prior to the preparation steps.

3. Ensure that dip-coated samples are dry after the final preparation step.

4. Consider the simplest preparation scheme. As an example, for the investigation of highlighter ink, just draw a dot on the substrate surface.

\section{Transfer of samples into DINeC mass spectrometer}

1. Transfer of samples from ambient conditions into the evacuated DINeC chamber

1. Vent the load-lock system.

2. Open the load-lock and mount the sample holder.

3. Close the load-lock and pump down the load-lock chamber to a pressure below $2 \times 10^{-5} \mathrm{mbar}$.

4. Open the valve to the DINeC chamber and transfer the sample holder with the transfer rod to the main manipulator. Attach the sample holder to the manipulator.

5. Retract the transfer rod and close the valve between load-lock and DINeC chamber. 
2. Transfer of samples from vacuum into the evacuated DINeC chamber

1. Use a transportable vacuum container, which can be attached to the CF40 flange of the DINeC chamber. Transfer the samples, which were prepared in vacuum, with this container without breaking the vacuum. Ensure that the samples are mounted on a sample holder compatible with the manipulator used in the DINeC system.

2. Attach the transportable vacuum container to the CF40 flange and pump down the volume between container and DINeC chamber.

3. Once the pressure has dropped below $2 \times 10^{-5} \mathrm{mbar}$, open the gate valves to the $\mathrm{DINeC}$ chamber and the transportable vacuum container and transfer the sample into the DINeC chamber onto the manipulator using a wobble stick or another transfer system with more than $50 \mathrm{~cm}$ linear movement.

4. Retract the transfer system and close the two gate valves.

\section{Preparation of gas mixture}

1. Prepare a mixture of approx. $3 \% \mathrm{SO}_{2}$ in helium by first evacuating the gas cylinders of the gas mixing system for 10 min

2. Fill the cylinders with $\mathrm{SO}_{2}$ until a pressure of 1 bar is reached.

3. Further fill the cylinders with helium until a total pressure of 30 bar is reached.

CAUTION: When using $\mathrm{SO}_{2}$, the respective safety precautions such as storing $\mathrm{SO}_{2}$ cylinders in designated gas cabinets have always to be fulfilled.

\section{Preparation of DINeC mass spectrometer}

1. Open the valve between the gas cylinder and the nozzle. Adjust the pressure of the $\mathrm{SO}_{2} / \mathrm{He}$ gas mixture at the outline of the gas mixing system to 15 bar.

2. Set the position of the manipulator to the reference sample's position.

3. For measuring cationic mass spectra, set the sample and grid bias to +40 and $+7 \mathrm{~V}$, respectively.

4. To drive the pulsed nozzle and the ion trap mass spectrometer, set the external function generator to $2 \mathrm{~Hz}$. With the delay generator, set the time delay $\Delta \mathrm{t}_{\mathrm{n}}$ between the Clear trap signal from the ion trap and the trigger signal for the pulsed nozzle to $5 \mathrm{~ms}$.

5. In the control software, adjust the following parameters by pressing the respective buttons or typing the respective values in the Mode page of the main dialogue window: Scan Mode: Enhanced resolution, Range: m/z 50 - 3000, Accu-time: 0.1 ms, Average: 10 cycles, Polarity: positive for the measurement of cationic mass spectra

NOTE: For measuring anionic spectra, the sample and grid bias have to be negative with respect to ground, Polarity has to be switched to Negative in the control software.

\section{Measurement of mass spectra}

1. Once a pressure below $3 \times 10^{-6} \mathrm{mbar}$ has been reached in the DINeC chamber, the measurement can be started. Start the measurement by first pressing Stand by and then pressing Operate in the control software. Start recording the measurements pressing the Play button.

2. Measure a test spectrum from a reference sample such as angiotensin II for about $300 \mathrm{~s}$. Follow the time dependence of the signal using the Chromatogram set to the respective $\mathrm{m} / \mathrm{z}$ value. Optimize signal intensity by adjustment of the time delay $\Delta \mathrm{t}_{\mathrm{n}}$ between the Clear trap signal and the signal triggering the pulsed nozzle.

3. Move the manipulator to the position of the sample to be measured. Optimize signal intensity by adjustment of the sample position within the sample holder plane.

4. Acquire mass spectra over the time span of interest. Modify the experimental parameters such as sample temperature or background pressure in the chamber according to the details of the experiment. Continue to take mass spectra when varying the experimental parameters.

\section{Data evaluation}

1. After the measurement is finished, load the respective data set in the Data Analysis program. Select the time span of interest in the chromatogram with the right button of the mouse. The averaged spectrum will be displayed in a separate window.

2. Export the spectrum as a data file for further processing in a program of choice. 


\section{Representative Results}

In the following, two examples for the real time application of DINeC-MS are presented. Figure 4 shows the change of the mass spectrum obtained from angiotensin II when the sample is heated to approx. $140^{\circ} \mathrm{C}$. When the final temperature is reached (Figure 4B, Figure 4E), the spectrum is characterized by an additional peak indicating the loss of an $\mathrm{H}_{2} \mathrm{O}$ entity $(\mathrm{m} / \mathrm{z}=1029)$. When keeping the sample at that temperature, further decomposition of the angiotensin II molecules is observed (Figure 4C), including the loss of one of the terminal amino acid units, aspartic acid (peak at $m / z=932$, Figure 4D). Quantitative analysis of the data allows to evaluate the underlying reaction kinetics (Figure 4E). In particular, Figure 4E demonstrates that the entity with $\mathrm{m} / \mathrm{z}=1029$ is an intermediate which further decomposes into smaller fragments as the intensity first increases and then decreases. The concomitant rate constants are thus in the same order of magnitude.

As a second example, the investigation of hydrogen/deuterium exchange in angiotensin $\mathrm{I}^{22}$ is illustrated in Figure 5. Upon exposure of the angiotensin sample to $\mathrm{D}_{2} \mathrm{O}$ in the DINeC chamber ( $p_{\mathrm{D} 2 \mathrm{O}}=10^{-4} \mathrm{mbar}$ ), the isotopic pattern of angiotensin II is broadened and shifted towards higher $\mathrm{m} / \mathrm{z}$ values indicating exchange of $\mathrm{H}$ by $\mathrm{D}$ atoms. The process is fast during the first $60 \mathrm{~s}$ but significantly slows down in the further course of the experiment: The isotope pattern in Figure 5B covers a wide $\mathrm{m} / \mathrm{z}$ range (approx. $15 \mathrm{~m} / \mathrm{z}$ units). When we define the degree of deuteration $d$ as the number of exchanged $\mathrm{H}$ atoms in a molecule, values of $d$ between $d=0$ to $d=13$ can be extracted from the spectrum. In Figure 5C, the isotope pattern is again reduced in width. This observation can be attributed to the strongly reduced intensity of the peaks which are associated with the lowest degrees of deuteration. In Figure 5D, the spectrum is shown for even longer reaction times. The covered $\mathrm{m} / \mathrm{z}$ range remains almost the same but the center of mass of the spectrum still shifts slowly towards increasing $\mathrm{m} / \mathrm{z}$ values. For long exposure times, a part of the molecules reaches the highest degree of deuteration, $d_{\max }=17$. It corresponds to the maximum number of exchangeable $\mathrm{H}$ atoms, given by the number of $\mathrm{H}$ atoms bound to functional groups such as carboxylic acids or amine groups.

Already from the temporal evolution of the spectra, it can be deduced that $\mathrm{H} / \mathrm{D}$ exchange takes place with different rate constants. For a quantitative description of this observation, the mean degree of deuteration $\bar{d}$ is plotted in Figure $\mathbf{5 E}$ as a function of time. Inspection of the experimental results (symbols) reveals three different regimes: a fast increase of $\bar{d}$ operative for $t<50 \mathrm{~s}$, an intermediate regime for $50 \mathrm{~s}<t<$ $200 \mathrm{~s}$, and a slow but almost continuous increase for $t>200 \mathrm{~s}$. The experimental results were simulated by means of Monte Carlo simulations; pseudo-first-order reaction kinetics with reaction constants $k_{\mathrm{i}}$ were assumed for the H/D exchange at the functional groups of the molecules investigated $^{22}$. A good agreement between simulations and experimental results in all three regimes was only obtained when at least three different rate constants $k_{\mathrm{i}}$ for the H/D exchange in angiotensin II molecules were applied. In Figure 5F,G, the kinetics as deduced from fitting the experimental isotope patterns by the sum of isotope patterns for different degrees of deuteration (Figure 5A to Figure 5D) are shown. The good agreement between experimental data and simulations as well as the very different exchange rates for low and high degrees of deuterations are clearly observed. By comparison with different oligopeptides such as hexaglycine, the fast exchange rates were attributed to the explicit functional groups, whereas the slow exchange rates were associated with the amide groups of the peptide's backbone ${ }^{22}$.

Whereas these first two examples were measured with micrometer-thick angiotensin II samples, Figure 6 shows the results as obtained from submonolayer coverage of angiotensin II on gold samples as prepared by means of electrospray ion-beam deposition (ES-IBD) $)^{23}$. A linear dependence of the signal intensity on amount of substance is observed over 3 orders of magnitude, the lowest amount of substance detected corresponds to $0.1 \%$ of a monolayer of angiotensin II molecules on the gold surface. H/D exchange experiments as shown in Figure $\mathbf{5}$ were also performed with angiotensin II on gold in the submonolayer regime ${ }^{23}$.

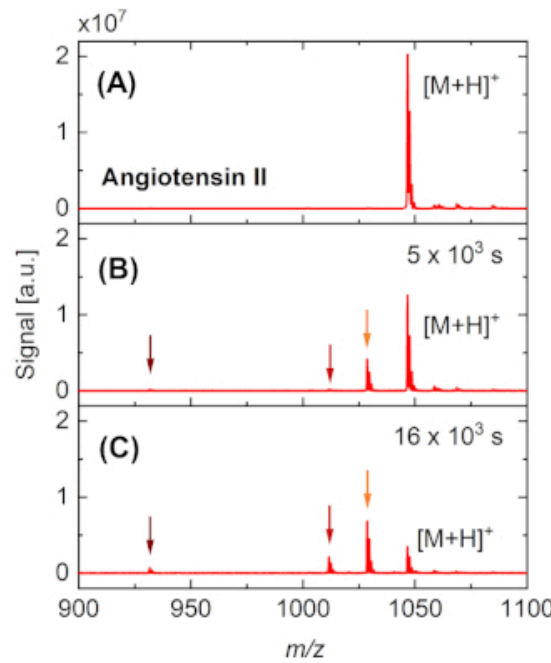

(D)
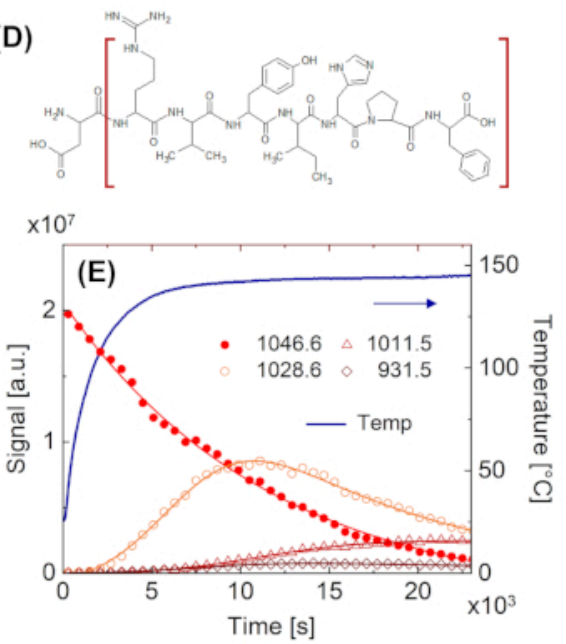

Figure 4: Real-time observation of thermal degradation of angiotensin II. (A-C) Mass spectra as obtained after cluster-induced desorption/ ionization from an angiotensin II sample. (A) Fresh sample at RT. (B) Sample heated to approx. $140{ }^{\circ} \mathrm{C}$. In addition to the peak at $m / z=1047$, which is associated with the intact molecule $[\mathrm{M}+\mathrm{H}]^{+}$, peaks at $\mathrm{m} / \mathrm{z}=932,1012$, and 1029 appear (indicated by arrows). (C) The latter peaks increase and the main peak decreases with time when keeping the sample at elevated temperature. (D) Structural formula of angiotensin II indicating the fragment (brown brackets) which leads to the appearance of the peak at $\mathrm{m} / \mathrm{z}=932$ by loss of one amino acid unit (aspartic acid). (E) Time dependence of sample temperature and the intensity of the main peaks indicated in the plots (A) to (C). Solid lines are guides to the eye. Please click here to view a larger version of this figure. 

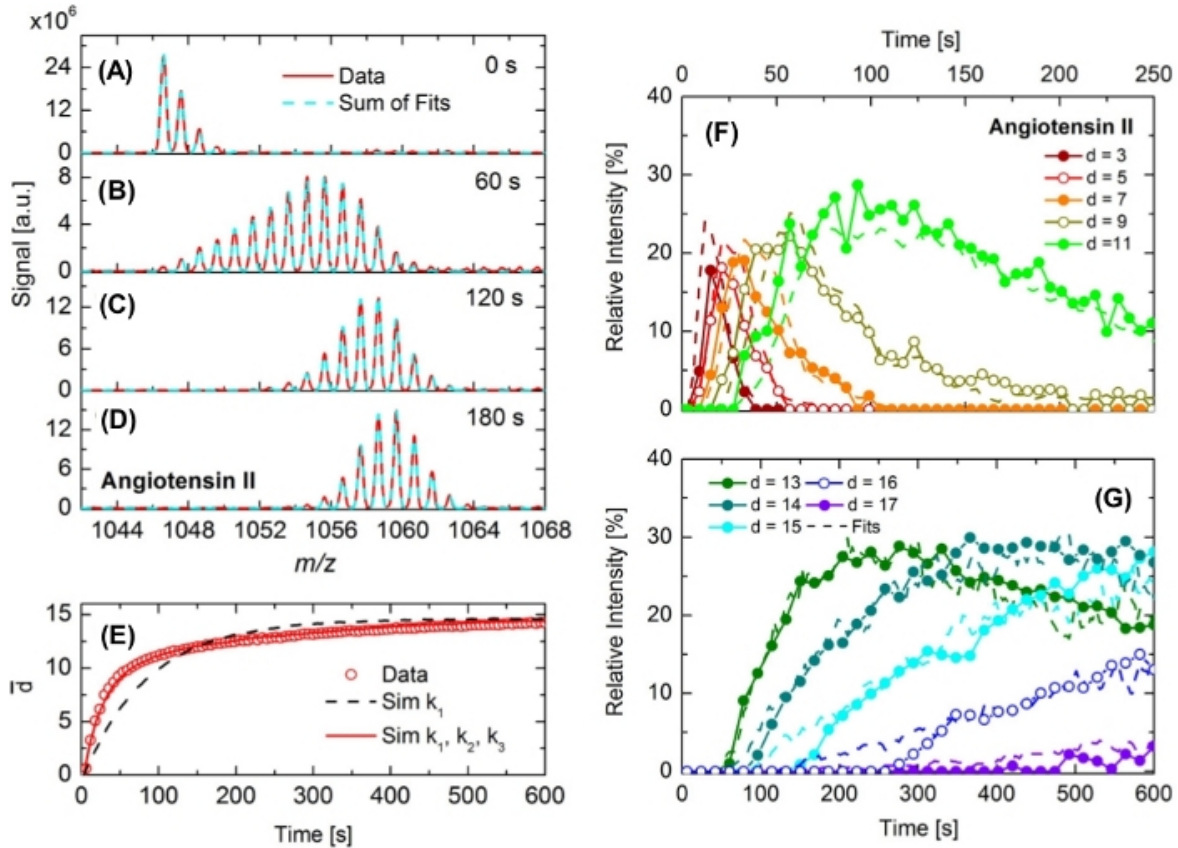

Figure 5: Real-time observation of H/D exchange in angiotensin II. (A-D) Cationic mass spectra of angiotensin II as obtained by means of DINeC-MS. Due to H/D exchange, the isotope pattern broadens and shifts towards higher $\mathrm{m} / \mathrm{z}$ values in (B) to (D) when compared to the isotope pattern of the undeuterated species shown in (A). Red lines are data, dashed cyan lines are fits to the data taking into account different degrees of deuteration. (E) Average degree of deuteration $\bar{d}$ as a function of time as deduced from the experiments (open dots). In addition, $\bar{d}$ as a function of time deduced by means of Monte Carlo simulations is shown. Black dashed curve: simulations taking into account one rate constant $\left(k_{1}\right)$; red curve: taking into account three rate constants $\left(k_{1}, k_{2}, k_{3}\right)$. $(\mathbf{F}, \mathbf{G})$ Relative signal intensities of selected degrees of deuteration of angiotensin II (symbols + solid lines) as a function of time together with the corresponding results of the Monte Carlo simulations (dashed lines). This figure has been modified from reference 22. Please click here to view a larger version of this figure.

(A)

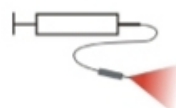

Preparation: ES-IBD

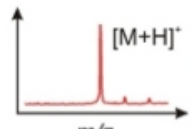

$m / 2$
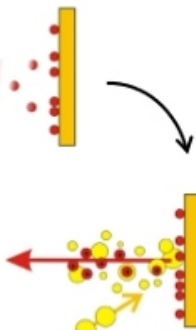

Analysis: DINeC-MS

(B)

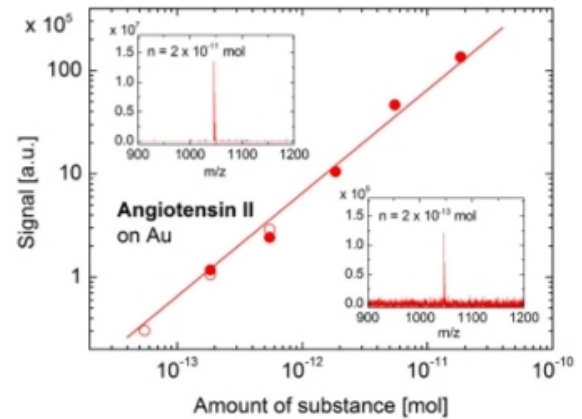

Figure 6: Application of DINeC-MS to angiotensin II on gold in the submonolayer regime. (A) Schematic representation of the combination of ES-IBD for deposition and DINeC-MS for mass spectrometry of isolated angiotensin II molecules in the submonolayer regime. (B) Dependence of signal intensity on amount substance deposited on the sample as obtained from two independent sets of data (filled and open symbols). Insets: DINeC mass spectra as obtained from samples on which an amount of substance was deposited as indicated. This figure has been modified from reference 23. Please click here to view a larger version of this figure.

\section{Discussion}

In many studies performed so far, a high sensitivity of DINeC-MS on various substances has been demonstrated. Indeed, this allows measurements of analytes down to an amount of substance in the femtomole regime ${ }^{11}$. Due to this high sensitivity, sample preparation, in particular substrate cleaning, has to be performed with highly pure chemicals in order to avoid contamination in the DINeC mass spectra. As it is the case for many analysis techniques, a proper background measurement from a blank substrate helps to separate peaks from the analyte and peaks which have their origin in substrate/sample preparation.

Although we have shown that the ionization probability of a given analyte molecule is not strongly influenced by the presence of co-adsorbates or co-constituents in mixed samples ${ }^{17,24}$, the ionization probability may vary from substance to substance ${ }^{13}$. Thus, it is even more important to work under clean conditions as contaminants, depending on their ionization probability, may contribute to the signal much stronger than the analyte. Preformed ions (e.g., as found in the case of many dye molecules), or molecules with functional groups which show a clear tendency towards proton uptake or deprotonation (i.e., bases or acids), typically show high ionization probability in DINeC-MS. If no such functional group is present in the analyte, ionization probability can be low. The samples then may be treated by ionizing agents such as trifluoro acid (e.g., by exposure of the sample to the vapor pressure of the ionizing agent). 
The representative results discussed in Figure $\mathbf{4}$ and Figure $\mathbf{5}$ demonstrate the applicability of DINeC-MS for real-time investigations of chemical reactions by means of mass spectrometry. Figure 6 illustrates the submonolayer-sensitivity of the method. If the two properties are combined, chemical reactions on surfaces and their products can be followed in real time ${ }^{23}$. This can be in particular of interest for so called "on-surface synthesis" which leads to the assembly of macromolecular structures on surfaces $3,33,34,35,36$. In the current set-up, the observation of such surface reactions is possible on surfaces with lower reactivity such as gold ${ }^{23}$ and other noble metals; the experiments are more difficult to be performed on highly reactive surfaces such as silicon surfaces ${ }^{37}$, as the base pressure in the desorption chamber is in the $10^{-7}$-mbar-range. Current activities address this limitation and a UHV-compatible DINeC apparatus is being built up. In the case of reactive surfaces, the interaction between $\mathrm{SO}_{2}$ and the substrate surface has to be tested prior to the measurements of surface adsorbates and surface reactions.

As the cluster beam is neutral, it cannot be focused. Beam size on the sample is thus given by the geometry of the set-up and orifice of the skimmer in use; typical values for the beam diameter on the sample is one to several millimeters. As a result, imaging by scanning the sample is possible only with very low resolution. On the other hand, given by the high ionization probability ${ }^{13}$, DINeC efficiently makes use of the desorbed molecules. Thus, a combination of DINeC-MS and an ion-imaging detector ${ }^{38}$ seems to be highly attractive.

\section{Disclosures}

The authors declare that they have nothing to disclose.

\section{Acknowledgments}

The authors acknowledge financial support from the Helmholtz International Center for FAIR (HICforFAIR) and the Helmholtz Graduate School for Hadron and Ion Research (P.S.). The authors thank Prof. Rauschenbach (University of Oxford) and his team for fruitful collaboration on combined ES-IBD/DINeC experiments.

\section{References}

1. Vickerman, J. C., Gilmore, I. Surface Analysis: The Principle Techniques. 2nd ed.; John Wiley \& Sons: New York (2009).

2. Reutzel, M., Münster, N., Lipponer, M.A., Länger, C., Höfer, U., Koert, U., Dürr, M. Chemoselective Reactivity of Bifunctional Cyclooctynes on $\mathrm{Si}(001)$. Journal of Physical Chemistry. C 120, 26284-26289 (2016).

3. Grill, L., Dyer, M., Lafferentz, L., Persson, M., Peters, M., Hecht, S. Nano-architectures by covalent assembly of molecular building blocks. Nature Nanotechnol. 2, 687-691 (2007).

4. Stutzmann, M.; Garrido, J. A., Eickhoff, M., Brandt, M. S. Direct biofunctionalization of semiconductors: A survey. Physica Status Solidi A. 203, 3424-3437 (2006).

5. Adler-Abramovich, L., Gazit, E. The physical properties of supramolecular peptide assemblies: from building block association to technological applications. Chemical Society Reviews. 43, 6881-6893 (2014).

6. Vickerman, J. C., Briggs, D. TOF-SIMS: Materials Analysis by Mass Spectrometry. 2nd ed., IM Publications: Chichester, UK (2013).

7. Winograd, N. The magic of cluster SIMS. Analytical Chemistry. 77, 142A-149A (2005).

8. Ichiki, K., Ninomiya, S., Nakata, Y., Honda, Y., Seki, T., Aoki, T., Matsuo, J. High Sputtering Yields of Organic Compounds by Large Gas Cluster lons. Applied Surface Science. 255, 1148-1150 (2008).

9. Mochiji, K., Hashinokuchi, M., Moritani, K., Toyoda, N. Matrix-free Detection of Intact lons from Proteins in Argon-Cluster Secondary lon Mass Spectrometry. Rapid Communications in Mass Spectrometry. 23, 648-652 (2009).

10. Yokoyama, Y., Aoyagi, S., Fujii, M., Matsuo, J., Fletcher, J. S., Lockyer, N. P., Vickerman, J. C., Passarelli, M. K., Havelund, R., Seah, M. P. Peptide Fragmentation and Surface Structural Analysis by Means of ToF-SIMS Using Large Cluster lon Sources. Analytical Chemistry. 88, 3592-3597 (2016).

11. Gebhardt, C. R., Tomsic, A., Schröder, H., Dürr, M., Kompa, K.L. Matrix-Free Formation of Gas-Phase Biomolecular lons by Soft ClusterInduced Desorption. Angewandte Chemie, International Edition. 48, 4162- 4165 (2009).

12. Baur, M., Lee, B.J., Gebhardt, C. R., Dürr, M. Soft Clusterinduced Desorption and lonization of Biomolecules - Influence of Surface Load and Morphology on Desorption Efficiency. Applied Physics Letters. 99, 234103 (2011).

13. Lee, B.J., Baur, M., Gebhardt, C. R., Dürr, M. Quantification of the Ionization Probability During Desorption/lonization of Oligopeptides Induced by Neutral Cluster Impact. Rapid Communications in Mass Spectrometry. 27, 1090-1094 (2013).

14. Lee, B.J., Gebhardt, C. R., Schröder, H., Kompa, K.L., Dürr, M. Observation of lonic Desorption Channels in Cluster-induced Desorption of Alkali Halides - Influence of Surface Electronic Properties and Surface Configuration. Chemical Physics Letters. 556, 77-81 (2013).

15. Baur, M., Gebhardt, C. R., Dürr, M. Desorption/Ionization Induced by Neutral Cluster Impact as a Soft and Efficient lonization Source for lon Trap Mass Spectrometry of Biomolecules. Rapid Communications in Mass Spectrometry. 28, 290-296 (2014).

16. Kley, C. S., Dette, C., Rinke, G., Patrick, C.E., Čechal, J., Jung, S.J., Baur, M., Dürr, M., Rauschenbach, S., Giustino, F., Stepanow, S., Kern, K. Atomic-Scale Observation of Multiconformational Binding and Energy Level Alignment of Ruthenium-Based Photosensitizers on $\mathrm{TiO}_{2}$ Anatase. Nano Letters. 14, 563-569 (2014).

17. Portz, A., Aoyagi, S., Dürr, M. Soft depth-profiling of mixed peptide/lipid samples by means of cluster induced desorption/ionization mass spectrometry - high depth resolution and low matrix effect. Biointerphases. 13, 03B405 (2018).

18. Portz, A., Baur, M., Gebhardt, C. R., Frank, A. J., Neuderth, P., Eickhoff, M., Dürr, M. Influence of the Cluster Constituents' Reactivity on the Desorption/lonization Process Induced by Neutral $\mathrm{SO}_{2}$ Clusters. Journal of Chemical Physics. 146, 134705 (2017).

19. Schneider, P., Dürr, M. Cluster-induced desorption investigated by means of molecular dynamics simulations - Microsolvation in clusters of polar and non-polar constituents. Journal of Chemical Physics. 150, 214301 (2019).

20. Karas, M., Hillenkamp, F. Laser Desorption Ionization of Proteins with Molecular Masses Exceeding 10000 Daltons. Analytical Chemistry. 60, 2299-2301 (1988).

21. Buchberger, A.R., DeLaney, K., Johnson, J., Li, L. Mass Spectrometry Imaging: A Review of Emerging Advancements and Future Insights Analytical Chemistry. 90, 240-265 (2018). 
22. Portz, A., Gebhardt, C. R., Dürr, M. Real-Time Investigation of the H/D Exchange Kinetics of Porphyrins and Oligopeptides by Means of Neutral Cluster-Induced Desorption/lonization Mass Spectrometry. Journal of Physical Chemistry B. 121, 11031-11036 (2017).

23. Portz, A., Baur, M., Rinke, G., Abb, S., Rauschenbach, S., Kern, K., Dürr, M. Chemical Analysis of Complex Surface-Adsorbed Molecules and Their Reactions by Means of Cluster-Induced Desorption/lonization Mass Spectrometry. Analytical Chemistry. 90, 3328 (2018).

24. Portz, A., Baur, M., Gebhardt, C. R., Dürr, M. Mass Spectrometry of Oligopeptides in the Presence of Large Amounts of Alkali Halides Using Desorption/lonization Induced by Neutral Cluster Impact. Biointerphases. 11, $02 A 316$ (2016).

25. Shard, A. G., Spencer, S. J., Smith, S. A., Havelund, R., Gilmore, I. S. International Journal of Mass Spectrometry. 377, 599-609 (2015).

26. Nakano, S., Yamagishi, T., Aoyagi, S., Portz, A., Dürr, M., Iwai, H., Kawashima, T. Evaluation of Matrix Effects on TOF-SIMS Data of Leuenkephalin and DOPC Mixed Samples. Biointerphases. 13, 03B403 (2018).

27. Heep, J., Tuchecker, P. H. K., Gebhardt, C. R., Dürr, M. Coupling of planar chromatography to mass spectrometry. ACS Omega. 4 , 22426-22430 (2019).

28. Morlock, G., Schwack, W., Coupling of planar chromatography to mass spectrometry, Trends in Analytical Chemistry. 29, 1157-1171 (2010).

29. Cheng, S.C., Huang, M.Z., Shiea, J., Thin layer chromatography/mass spectrometry, Journal of Chromatography A. 1218, $2700-2711$ (2011).

30. Takats, Z., Wiseman, J.M., Gologan, B., Cooks, R.G. Mass spectrometry sampling under ambient conditions with desorption electrospray ionization. Science. 306, 471 (2004).

31. Cooks, R. G., Ouyang, Z., Takats, Z., Wiseman, J.M. Ambient mass spectrometry. Science. 311, 1566 (2006).

32. Dürr, M., Gebhardt, C. Ion generation in mass spectrometers by cluster bombardment, US Patent 926322. B2. (2019).

33. Lindner, R., Kühnle, A. Bottom-up Assembly of Molecular Wagons on a Surface. ChemPhysChem. 16, 1582-1592 (2015).

34. Dong, L., Liu, P.N., Lin, N. Bottom-up Assembly of Molecular Wagons on a Surface. Accounts of Chemical Research. 48, 2765-2774 (2015).

35. Björk, J. Reaction mechanisms for on-surface synthesis of covalent nanostructures. Journal of Physics: Condensed Matter. 28, 083002 (2016).

36. Rauschenbach, S., Rinke, G., Gutzler, R., Abb, S., Albarghash, A., Le, D., Rahman, T. S., Dürr, M., Harnau, L., Kern, K. Two-Dimensional Folding of Polypeptides into Molecular Nanostructures at Surfaces. ACS Nano. 11, 2420-2427 (2017).

37. Dürr, M., Höfer, U. Dissociative adsorption of molecular hydrogen on silicon surfaces. Surface Science Reports. 61, 465-526 (2006).

38. Zhang, J., Franzreb, K., Aksyonov, S.A., Williams, P. Mass Spectra and Yields of Intact Charged Biomolecules Ejected by Massive Cluster Impact for Bioimaging in a Time-of-Flight Secondary Ion Microscope. Analytical Chemistry. 87, 10779-10784 (2015). 